\title{
The Role of Endocannabinoid System in Sleep Deprivation- Induced Psychosis-Like Symptoms through Hampering Prepulse Inhibition; A Hypothesis
}

Mohammad Nami 1,2,3,4*, Ali-Mohammad Kamali 1,2, Milad Kazemiha 1,2, Rao kosagisharaf 4, Sarbi Derman ${ }^{5}$

1Department of Neuroscience, School of Advanced Medical Sciences and Technologies, Shiraz University of Medical Sciences, Shiraz, Iran

2Dana Brain Health Institute, Iranian Neuroscience Society-Fars Chapter, Shiraz, Iran

${ }^{3}$ Academy of Health, Senses Cultural Foundation, Sacramento, CA, USA

${ }^{4}$ Neuroscience Center, INDICASAT-AIP, Panama City, Republic of Panama

${ }^{5}$ American Hospital, Koc Foundation, Istanbul, Turkey

*Corresponding Author: Mohammad Nami MD, PhD. Head of the Department of Neuroscience, School of Advanced Medical Sciences and Technologies, Shiraz University of Medical Sciences, Shiraz, Iran. torabinami@sums.ac.ir

\begin{abstract}
The interaction between endocannabinoid (eCB) system with in key brain structures such as hippocampus, amygdala and prefrontal cortex and sleep deprivation (SD)-induced psychosis has been less studied. The present hypothesis revolves around the question whether altered chemical dynamics within the eCB system with the resultant impact on cannabinoid receptors in key cortical hubs would impact SD-induced psychosis-like symptoms. Having this investigated research is expected to pave the path towards identifying newer drug targets namely for schizophrenia.
\end{abstract}

Keywords: endocanabinoid system; sleep deprivation; animal models; psychosis-like symptoms; drug development

\section{The hypothesis}

With today's competitive life, sleep deprivation (SD) has been turned to a constantly-growing issue leaving negative impact on overall health at personal and societal levels(1). Overwhelming stress, poor sleep hygiene habits, shift works, psycho-behavioral or medical problems, dysregulated endocrine system, the use of stimulant medications and substance abuse are among the factors which have collectively contributed to the increasing prevalence of sleep insufficiency worldwide(2, 3).

When becomes a chronic issue, the clinical consequences of untreated sleep disorders are substantial wherein hypertension, ischemic heart disease, heart failure, cerebrovascular accidents, obesity, psychiatric conditions and cognitive predicaments become underway(4). Some recent studies have proposed acute SD as an experimental animal model for psychosis(5). The state of total SD has been documented to result in perceptual disturbances such as hallucinations and illusions, thought disorder, delusions, and fear-related symptoms 
in human(5). Additionally, SD is known to cause psychosis-like reduction in prepulse inhibition (PPI) in a rat model(6).

Recently, longitudinal and postmortem studies have shown that the endocannabinoid (eCB) system might be involved in neuropsychiatric disorders like schizophrenia(7). One the other hand, cannabis consumption may induce a psychotic state in normal individuals and worsen psychotic symptoms of schizophrenia patients(8). The endocannabinoid (eCB) system is known to be involved in psychoactive effects of cannabis. This system contributes to a variety of physiological processes such as appetite, pain perception, mood regulation, and cognition(9). As for appetite for instance, we know rats which undergo SD tend to have an increased food intake and this might be in some ways related to the effect of SD left on the eCB system(10).

The eCB system hosts endogenous cannabinoids such as anandamide ( $N$ arachidonoylethanolamide, (AEA), 2-arachidonoylglycerol (2-AG) and its structural analogue 2oleoylglycerol (2-OG). It also nests the enzymes that synthesize and degrade endocannabinoids, including fatty acid amide hydrolase (FAAH) or monoacylglycerol lipase (MAGL) as well as CB1 and CB2(11). Recent findings propose that dysregulation in the eCB system may underpin the pathophysiology of schizophrenia(8). As such, therapeutic approaches in schizophrenia may at least partly depend on modulating eCB dynamics. For instance, cannabinoid drugs impair PPI in an animal model of schizophrenia(12, 13).

Continuous exposure to tetrahydrocannabinol (THC) has been shown to upregulate the eCB system, leading to long-lasting neurobiological changes in various regions of the brain(14). Also after sleep restriction, increase in endocannabinoid level is amplified(15) and levels of the eCB system compound 2-oleoylglycerol (2-OG) increased(16). By acting on cannabinoid receptors, THC also influences the release of neurotransmitters involved in the pathophysiology of schizophrenia, including dopamine and glutamate(17). As a result, heavy consumption of cannabis most often has harmful effects on an individual's health and can lead to the development of psychosis and schizophrenic-like symptoms $(18,19)$. The above hypothesized interplay is depicted in Figure 1.

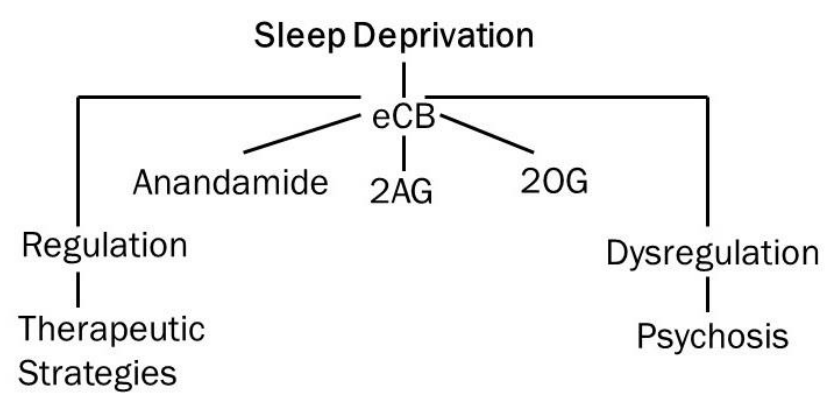

Figure 1. The hypothesized interplay between eCB system and its dysregulation in relation with sleep deprivation and psychosis. eCB: endocannabinoid system, 2AG: 2-arachidonoylglycerol, 20G: 2-oleoylglycerol

Despite the above, the interaction between SD, psychosis, and eCB system has not been sufficiently studied yet. It is worth to investigate the mechanisms through which SD induces 
psychosis-like symptoms. The question whether total and paradoxical SD impair the PPI in rats and how eCB system is involved in such impairment needs to be spotlighted in future studies. To this aim, we hypothesize that measuring circulating levels of 2-AG, 2-OG and AEA, FAAH, MAGL and CB1 in the hippocampus, amygdala and PFC using real-time PCR and HPLC would potentially provide a clue. Research may be pursued by blocking the eCB system systemically after SD using eCB receptors antagonists to identify if SD still hampers the PPI. Continued research would also need to investigate if antipsychotic and cognitive enhancing drugs, such as cannabidiol and modafinil reverse the effects of SD upon PPI impairments.

\section{Conclusion}

What already known is that SD per se is considered as a validated model for schizophrenia(5, 6). Meanwhile, what is yet to be explored is that how eCB system is involved in the process of psychosis-like behaviors induced by SD and what the mostly involved neurobiochemical elements are within the system. Having this addressed, the outcome may potentially provide novel insights into the eCB neurobiochemical dynamics implicated in the pathogenesis of schizophrenia. While other potential culprits such as genetic predisposition play key parts in the pathogenesis of schizophrenia, there are still much to be addressed with regards to the neurbiochemical interplay between sleep loss, schizophrenia and eCB system.

The present paper hypothesizes that eCB system might be critically involved in regulating the effects of SD on impairing PPI and the subsequent psychosis-like symptoms. The identification of eCB molecular pathways implicated in the deleterious psychiatric effects of SD in an animal model could potentially yield new targets for the development of more effective drugs.

\section{Conflict of Interest}

Authors declared no conflict of interest upon preparation of the resent work.

\section{Acknowledgement}

Authors are thankful to the National Science System SNI of SENACYT, Panama for their support.

\section{Reference}

1. Skaer TL. Sleep deprivation and economic burden. Sleep Deprivation and Disease: Springer; 2014. p. 269-79.

2. Stores G. Sleep disorders in general and in adolescence. The journal of family health care. 2009;19(2):51-3.

3. Torabi-Nami M. Sleep hygiene the gateway for efficient sleep: A brief review. WebmedCentral BRAIN. 2011;2.

4. Torabi-Nami M, Mehrabi S, Borhani-Haghighi A, Derman S. Withstanding the obstructive sleep apnea syndrome at the expense of arousal instability, altered cerebral autoregulation and neurocognitive decline. Journal of integrative neuroscience. 2015;14(02):169-93.

5. Petrovsky N, Ettinger U, Hill A, Frenzel L, Meyhöfer I, Wagner M, et al. Sleep deprivation disrupts prepulse inhibition and induces psychosis-like symptoms in healthy humans. Journal of neuroscience. 2014;34(27):9134-40. 
6. Meyhöfer I, Kumari V, Hill A, Petrovsky N, Ettinger U. Sleep deprivation as an experimental model system for psychosis: effects on smooth pursuit, prosaccades, and antisaccades. Journal of Psychopharmacology. 2017;31(4):418-33.

7. Fervaha G, Zakzanis KK, Foussias G, Graff-Guerrero A, Agid O, Remington G. Motivational deficits and cognitive test performance in schizophrenia. JAMA psychiatry. 2014;71(9):1058-65.

8. Ksir C, Hart CL. Cannabis and psychosis: a critical overview of the relationship. Current psychiatry reports. 2016;18(2):12.

9. Mechoulam R, Parker LA. The endocannabinoid system and the brain. Annual review of psychology. 2013;64:21-47.

10. Torabi-Nami M, Nasehi M, Zarrindast M-R. Sleep loss and the brain vulnerability to neurodegeneration: behavioral, biochemical and neuro-histopathological observations in a rat model. Excli Journal. 2013;12:347-72.

11. Sugiura T, Oka S, Gokoh M, Kishimoto S, Waku K. New perspectives in the studies on endocannabinoid and cannabis: 2-arachidonoylglycerol as a possible novel mediator of inflammation. Journal of pharmacological sciences. 2004;96(4):367-75.

12. Levin R, Peres FF, Almeida V, Calzavara MB, Zuardi AW, Hallak JEC, et al. Effects of cannabinoid drugs on the deficit of prepulse inhibition of startle in an animal model of schizophrenia: the SHR strain. Frontiers in pharmacology. 2014;5:10.

13. Brzózka MM, Fischer A, Falkai P, Havemann-Reinecke U. Acute treatment with cannabinoid receptor agonist WIN55212. 2 improves prepulse inhibition in psychosocially stressed mice. Behavioural brain research. 2011;218(2):280-7.

14. Renard J, Krebs M-O, Le Pen G, Jay TM. Long-term consequences of adolescent cannabinoid exposure in adult psychopathology. Frontiers in neuroscience. 2014;8:361.

15. Hanlon EC, Tasali E, Leproult R, Stuhr KL, Doncheck E, De Wit H, et al. Sleep restriction enhances the daily rhythm of circulating levels of endocannabinoid 2-arachidonoylglycerol. Sleep. 2016;39(3):653-64.

16. Bhutani S, Howard JD, Reynolds R, Zee PC, Gottfried J, Kahnt T. Olfactory connectivity mediates sleep-dependent food choices in humans. eLife. 2019;8.

17. Fernandez-Espejo E, Viveros M-P, Núñez L, Ellenbroek BA, De Fonseca FR. Role of cannabis and endocannabinoids in the genesis of schizophrenia. Psychopharmacology. 2009;206(4):531-49.

18. Malone DT, Hill MN, Rubino T. Adolescent cannabis use and psychosis: epidemiology and neurodevelopmental models. British journal of pharmacology. 2010;160(3):511-22.

19. D'Souza DC, Sewell RA, Ranganathan M. Cannabis and psychosis/schizophrenia: human studies. European archives of psychiatry and clinical neuroscience. 2009;259(7):413-31. 